\title{
Influence of different facets of internet addiction on subjective well-being in Malaysia: a comparison across ethnic groups
}

\begin{abstract}
Despite a growing awareness that internet addiction is becoming a significant public health issue, there is limited research on how multiple facets of internet addiction relates to the humanistic concepts of well-being, particularly those captured in Diener's three subjective well-being dimensions: life satisfaction, positive affect, and negative affect across ethnic groups. Thus, this study aimed to investigate the relationship between different facets of internet addiction and the three subjective well-being dimensions in Malaysia. The research work also sought to investigate if the relationship operates equivalently across ethnic groups. Malaysians ( $n=400,66.5 \%$ female; age in years $M=24.52, S D=5.7)$ completed Satisfaction with Life Scale and the PANAS. Structural equation modelling was used to examine these associations. Results showed that six facets of internet addiction, such as mood modification, impaired control, conflict, preoccupation, tolerance, and withdrawal, were significant predictors of lower subjective well-being on almost all scales across the total sample size. Invariances analyses also indicated that there were a few significant ethnic differences across these predictors for subjective well-being. The findings highlight the importance of efforts to reduce various characteristic symptoms of internet addiction to mitigate its harmful effects on subjective well-being across ethnic groups in Malaysia.
\end{abstract}

Keyword: Internet addiction; Subjective well-being; Ethnic groups; Malaysia; Multidimensional 